\title{
Should Let Them Go? Study on the Emergency Department Discharge of Patients Who Attempted Suicide
}

\author{
Heejun Shin ${ }^{1}$, Ho Jung Kim ${ }^{1}$, Shingyeom Kim², Sunjin $\mathrm{Choi}^{3}$, Heeju $\mathrm{Oh}^{3}$, and Bora $\mathrm{Lee}^{4}$ \\ ${ }^{1}$ Department of Emergency Medicine, Soonchunhyang University, Bucheon, Republic of Korea \\ ${ }^{2}$ Department of Psychiatry, Soonchunhyang University, Bucheon, Republic of Korea \\ ${ }^{3}$ Departement of Suicide Prevention, Bucheon Hospital of Soonchunhyang University, Bucheon, Republic of Korea \\ ${ }^{4}$ Department of Biostatistics, Clinical Trial Center, Soonchunhyang University, Bucheon, Republic of Korea
}

Objective The purpose of this study was to analyze the characteristics and factors of voluntary discharged patients after suicide attempt and analyze the effectiveness of follow-up measures.

Methods Total 504 adult patients aged 14 years and over, who visited a local emergency medical center from September 1, 2013 to December 31, 2015 were enrolled and retrospectively reviewed. We analyzed the relationship with voluntary discharge group (VDG) among basic characteristics, suicidal attempt variables, outcome variables related to suicide attempts, and treatment related variables comparing with normal discharge group (NDG).

Results Of the total 504 suicide attempts, three hundred eleven (61.7\%) patients were VDG and 193 (38.2\%) were NDG. The proportion of patients who completed the community service linkage were 18.7\% (36/193) in NDG, compared with 7.7\% (24/311) in VDG $(\mathrm{p}<0.05)$. In addition, the ratio of the patients who visited psychiatric outpatient department in NDG were $57.0 \%(110 / 193)$, more than four times as likely as $14.5 \%(45 / 311)$ in VDG $(\mathrm{p}<0.05)$.

Conclusion Over sixty percent of suicide attempters discharged against medical advice. Further various aspects of national supportive measures including strengthening case management service should be considered.

Psychiatry Investig 2018;15(6):638-648

Key Words Suicide, Analysis, Statistics, Prevention.

\section{INTRODUCTION}

A suicide attempter is a patient who has survived a suicide attempt. Persons with suicidal thoughts are estimated to be approximately 25 times more likely to attempt suicide than the general population. ${ }^{1,2}$ Both individual and social factors have been implicated in suicidal tendencies, as explained by Emile Durkheim in the late 19th century. ${ }^{3}$ Many suicide attempters come to the emergency department due to special circumstances, such as psychiatric problems, substance abuse (e.g., alcohol), and physical injury from previous suicide attempts. Since 2013, the Suicide Trial Case Management Team

Received: October 28, 2017 Revised: March 9, 2018

Accepted: April 15, 2018

$\triangle$ Correspondence: Ho Jung Kim, MD, PhD

Department of Emergency Medicine, Bucheon Hospital of Soonchunhyang University, 170 Jomaru-ro, Bucheon 14584, Republic of Korea

Tel: +82-32-621-5119, Fax: +82-32-621-6560, E-mail: khj995375@naver.com

(c) This is an Open Access article distributed under the terms of the Creative Commons Attribution Non-Commercial License (http://creativecommons.org/licenses/by$\mathrm{nc} / 4.0$ ) which permits unrestricted non-commercial use, distribution, and reproduction in any medium, provided the original work is properly cited. has been deployed as a national emergency center of the emergency management system of the Republic of Korea. However, although most suicide attempters are treated by a physician, most of them are still discharged voluntarily after completing their paperwork, contrary to medical advice. Voluntary discharge of suicidal patients is a problem in terms of the patient's protection, given that the percentage of patients involved in re-entry with the same diagnosis is higher than that of other patients. ${ }^{1}$ In addition, the domestic mental health service has adopted a policy that minimizes forced hospitalization and treatment of severely mentally ill patients; this policy is based on the recommendations of the United Nations Convention on the Rights of Persons with Disabilities that emphasizes the elimination of forced hospitalization and treatment. It is therefore difficult for emergency medical physicians to prevent voluntary discharge of suicide attempters. ${ }^{1}$

Among the Organization for Economic Co-operation and Development (OECD) countries with the highest suicide percentages from 1985-2001, Finland and Japan succeeded in decreasing their suicide percentages by conducting govern- 
ment suicide prevention projects and suicide comprehensive measures centered on "psychological autopsy". The government of the Republic of Korea also enacted relevant laws in 2012, established the Korean Suicide Prevention Center, and started an emergency department-based suicide attempt management project in 2013. ${ }^{1}$ In addition, the Korean Psychological Autopsy Center has been established for the purpose of identifying the cause of suicide death. ${ }^{1}$ Despite these efforts, the suicide prevalence in Korea is 27.3/100,000 of the general population, which is twice the OECD suicide average of $12.0 /$ 100,000 of the general population in 2014. ${ }^{1}$ Domestic efforts to reduce the suicide percentage have been initiated by the Korea government; however, as these programs are at their beginning, domestic studies on the discharge of suicide attempters are rare.

The purpose of this study was therefore to investigate the characteristics and factors related to the discharge of patients and the effectiveness of state-led case management for suicide attempts by hospital emergency departments.

\section{METHODS}

\section{Periods and subjects}

Among 506 adult patients $\geq 14$ years of age who visited the Soonchunhyang University Hospital Emergency Medical Center at Bucheon City, which is located in Gyeongi-do province in southern Korea, from September 1, 2013 to December 31, 2015, two patients were excluded from the study due to missing data about their medical severities. The medical records of 504 patients were then reviewed retrospectively.

\section{Key variables}

The statistical relationship between the major variables of the four categories (basic characteristics, suicide attempt-related variables, outcome variables related to suicide attempts, and treatment-related variables) and the types of discharges were investigated. Independent variables were defined as variables that were necessary to predict a voluntary discharge. The type of discharge of the attempted suicide victim admitted to the emergency department was classified into the voluntary discharge group (VDG) and normal discharge group (NDG). Dependent variables were defined as VDG and NDG. The methods of attempted suicide were categorized as ingested poisoning, briquette gas poisoning, hanging, wrist cutting/puncture wounds, death leaps, and other causes. The state of consciousness at presentation was classified as alert, verbal, painful, and unresponsive.

Medical severity was defined as the extent of a serious injury. The medical severity was based on the medical records prepared by the emergency department nurse and interviews of two case management service team members. We classified cases where intensive care was required due to the severity of the injury, even after emergency department treatment, as "high." After emergency department treatment, the patients who were admitted to the general ward or who needed medical attention even after discharge were classified as "moderate." If there was no physical damage or very mild (abrasion) physical damage usually treated with a simple dressing, the case was classified as "low." After emergency department discharge, the researcher completed the type of insurance by checking the medical records and classifying them as "health insurance," "ordinary type," "medical aid," and "others." Patients with a history of psychiatric disease were covered by health insurance and classified as "health insurance." Other suicide attempters in the emergency department were recorded as "ordinary type" and were not covered by insurance. However, if the patient agreed to psychiatric treatment in the emergency department and conducted a psychiatric consultation, "ordinary type" was changed to "health insurance." This was confirmed by reviewing the medical records of the authors after discharge of the patient and confirming the changed parts of the records. Even if the psychiatric consultation occurred in the emergency department, it was classified as "ordinary type" when the patient was not cooperating with the interview, when it was difficult to eliminate a diagnosis, or if psychiatric treatment was refused after discharge.

The classification of independent variables and dependent variables are listed below.

\section{Independent variables}

1) Baseline characteristics (demographic variables): sex, age, level of education, marital status, housemate, physical status, and the type of insurance.

2) Variables related to suicide attempts: drinking, a history of psychiatric disease, the method of the suicidal attempt, acknowledgment of the suicide attempt, and plans about a future suicide attempt.

3) Suicide attempt-related outcomes per type of discharge: awareness condition, medical severity, and plans for future suicide attempts.

4) Treatment modalities per type of discharge: medical request for neuropsychiatry (NP), case management service, links with community services, psychiatric treatment after the discharge, location of the discharge.

\section{Dependent variables: VDG and NDG}

\section{Research methods and statistical analyses}

We used descriptive statistics to determine how these four categories of independent variables listed below were related 
to the voluntary discharge.

(1) Baseline characteristics (demographic variables)

(2) Variables related to suicide attempts

(3) Variables of suicidal attempt-related outcomes

(4) Treatment-related variables

The chi-square test was used to compare the main variables of the above four categories from (1) to (4) according to the type of discharge.

(5) Frequencies of emergency department voluntary discharge of patients according to visit and discharge time differences

Simple descriptive statistics (frequency and percentage) were applied for category (5).

(6) Voluntary discharge outcomes according to univariate logistic regression analyses

Univariate logistic regression analyses were performed for all variables included in the four categories in the suicide attempt group. The variables associated with voluntary discharge and the extents of association with statistical significance were also later examined.

(7) Voluntary discharge outcomes according to multivariate logistic regression analyses

After constructing an initial model of multivariate logistic regression using statistically significant factors in univariate logistic regression, we selected variables by backward selection and examined the variables associated with voluntary discharge and their associated strengths of statistical significance.

The collected data were analyzed using the $\mathrm{R}$ 3.1.3 program (codename, "Smooth Sidewalk"), (Comprehensive R Archive Network at http://cran.r-project.org/). The chi-square test, and univariate and multivariate logistic regression analyses were used as the statistical methods. In the table, the percentages in parenthesis of the frequency of each variable, including the subgroups, are expressed as a percentage of the frequency of variables corresponding to total discharge, voluntary discharge, and normal discharge using the chi-square test. In the table of univariate and multivariate logistic regression analyses, the odds ratio (OR) with a 95\% confidence interval was expressed with a p-value for the voluntary discharge. Percentages were rounded off to two decimal places. A value of $\mathrm{p}<0.05$ was defined as statistically significant.

\section{Institutional review board}

This study was supported by Soonchunhyang University and approved by the Institutional Review Board Committee of Soonchunhyang University Bucheon Hospital (IRB No. 201708-019-002).

\section{RESULTS}

\section{Baseline characteristics (demographic variables)}

Age, level of education, marital status, and physical status were statistically significant in terms of baseline characteristics (Table 1). Of the total 504 suicide attempts, 311 (61.7\%) involved VDG patients and 193 (38.2\%) involved NDG patients. Although there was no statistically significant relationship between sex and the VDG, 211 (67.8\%) out of a total of 311 (100\%) VDG patients were female, which was more than twice as many as the $100(32.2 \%)$ males. The percentage of VDG patients $(104 / 311,33.4 \%)$ was higher than that of NDG patients $(48 / 193,24.8 \%)$ for patients $<20$ years of age, and the percentage of NDG patients $(73 / 193,37.8 \%)$ was higher than those of VDG patients $(86 / 311,27.7 \%)$ for patients $>50$ years of age $(\mathrm{p}<$ 0.05 ). Regarding the level of education or physical status, the percentages of unmeasured (non-responders) patients were $74.6 \%(232 / 311)$ and $44.4 \%(138 / 311)$, respectively, with the percentage of VDG patients higher than those of $37.3 \%$ (72/193) and $13.0 \%(25 / 193)$, respectively, in the NDG $(\mathrm{p}<0.05)$. The percentage of patients in the non-responder group with a level of education below high school was at least five times higher than that of university graduates in both the VDG and NDG $(\mathrm{p}<0.05)$. Regarding physical status, the percentage of the healthy group was the highest and the percentage of the acute disease group was the lowest for both the VDG and NDG, respectively $(\mathrm{p}<0.05)$. Regarding marital status, the percentage of the married group was the lowest compared with unmarried or married but without a spouse group in the VDG, whereas the percentage of the married group was the highest compared with those in the NDG $(\mathrm{p}<0.05)$.

\section{Variables related to suicide attempts}

Variables related to suicide attempts such as drinking, a history of psychiatric disease, the method of the suicidal attempt, acknowledgment about the suicidal attempt, and a plan for a future suicide attempt were all significantly associated with voluntary discharge $(\mathrm{p}<0.05)$ (Table 2$)$. In the VDG, the percentage of drinking patients was the highest compared with the percentage of non-drinking patients in the unmeasured group $(p<0.05)$ (Table 2). Except for the non-response group, patients with a psychiatric history were more likely to voluntarily discharge than those who were not in the VDG $(\mathrm{p}<0.05)$. In addition, the percentage of ingested poisoning was $51.8 \%$ (161/311), which was higher than other methods of suicide attempts in the VDG $(\mathrm{p}<0.05)$. The percentage of patients with a plan for a future suicidal attempt of the impulse group, except for the unmeasured group, was 46.6\% (145/311) compared with those with a plan for a future suicide attempt $[6.8 \%(21 / 311)]$ in the VDG, which was more than seven times 
Table 1. Baseline characteristics per type of discharge

\begin{tabular}{|c|c|c|c|c|}
\hline Variable & $\begin{array}{c}\text { Total } \\
(\mathrm{N}=504)\end{array}$ & $\begin{array}{l}\text { Voluntary discharge group } \\
\qquad(\mathrm{N}=311)\end{array}$ & $\begin{array}{l}\text { Normal discharge group } \\
\qquad(\mathrm{N}=193)\end{array}$ & p-value \\
\hline $\operatorname{Sex}(\%)$ & & & & 0.15 \\
\hline Male & $175(34.7)$ & $100(32.2)$ & $75(38.9)$ & \\
\hline Female & $329(65.3)$ & $211(67.8)$ & $118(61.1)$ & \\
\hline Age (year) (\%) & & & & 0.022 \\
\hline$<20$ & $42(8.3)$ & $29(9.3)$ & $13(6.7)$ & \\
\hline $20-29$ & $110(21.8)$ & $75(24.1)$ & $35(18.1)$ & \\
\hline $30-39$ & $92(18.3)$ & $53(17.0)$ & $39(20.2)$ & \\
\hline $40-49$ & $101(20.0)$ & $68(21.9)$ & $33(17.1)$ & \\
\hline $50-59$ & $90(17.9)$ & $55(17.7)$ & $35(18.1)$ & \\
\hline$\geq 60$ & $69(13.7)$ & $31(10.0)$ & $38(19.7)$ & \\
\hline Educational level (\%) & & & & $<0.001$ \\
\hline High school or less & $169(33.5)$ & $68(21.9)$ & $101(52.3)$ & \\
\hline$\geq$ College & $31(6.2)$ & $11(3.5)$ & $20(10.4)$ & \\
\hline No response & $304(60.3)$ & $232(74.6)$ & $72(37.3)$ & \\
\hline Marital status (\%) & & & & 0.007 \\
\hline Unmarried & $184(36.5)$ & $120(38.6)$ & $64(33.2)$ & \\
\hline Married & $167(33.1)$ & $87(28.0)$ & $80(41.5)$ & \\
\hline Married but without spouse & $153(30.4)$ & $104(33.4)$ & $49(25.4)$ & \\
\hline Housemate (\%) & & & & 0.679 \\
\hline Existence & $426(84.5)$ & $265(85.2)$ & $161(83.4)$ & \\
\hline None & $78(15.5)$ & $46(14.8)$ & $32(16.6)$ & \\
\hline Physical status (\%) & & & & $<0.001$ \\
\hline Healthiness & $186(36.9)$ & $102(32.8)$ & $84(43.5)$ & \\
\hline Acute disease & $13(2.6)$ & $5(1.6)$ & $8(4.1)$ & \\
\hline Chronic disease/disability & $142(28.2)$ & $66(21.2)$ & $76(39.4)$ & \\
\hline Unmeasured & $163(32.3)$ & $138(44.4)$ & $25(13.0)$ & \\
\hline Type of insurance (\%) & & & & 0.096 \\
\hline Health insurance & $277(55.0)$ & $181(58.2)$ & $96(49.7)$ & \\
\hline Medical aid & $26(5.2)$ & $18(5.8)$ & $8(4.1)$ & \\
\hline Ordinary type & $191(37.9)$ & $108(34.7)$ & $83(43.0)$ & \\
\hline Others & $10(2.0)$ & $4(1.3)$ & $6(3.1)$ & \\
\hline
\end{tabular}

Data were reported as frequency (percentage). p-values were calculated by chi-square test or Fisher's exact test as appropriate

higher than those who planned to commit suicide $(\mathrm{p}<0.05)$ (Table 2).

\section{Variables of suicide attempt-related outcomes}

Variables of suicide attempt-related outcomes were the awareness condition, medical severity, and future suicide attempt plans (Table 3). Of these, the remaining variables except for the awareness condition showed a significant relationship with voluntary discharge of the patient $(\mathrm{p}<0.05)$ (Table 3$)$. The lower the medical severity, the higher the percentage of voluntary discharge, and in cases of future suicide attempt plans, the percentage of patients who did not disclose their intentions was the highest in the VDG $(\mathrm{p}<0.05)$ (Table 3$)$.

\section{Treatment-related variables}

The variables related to the treatment of suicide attempts were medical requests for NP, case management service, links with community service, psychotherapy after discharge, and the location when discharged. All these variables showed a statistically significant relationship with voluntary discharge $(\mathrm{p}<0.05)$ (Table 4, Figure 1). Of a total of 504 suicide attempts, $311(61.7 \%)$ were discharged voluntarily from the emergency 
Table 2. Suicidal attempt related characteristics per type of discharge

\begin{tabular}{|c|c|c|c|c|}
\hline Variable & $\begin{array}{c}\text { Total } \\
(\mathrm{N}=504)\end{array}$ & $\begin{array}{l}\text { Voluntary discharge group } \\
\qquad(\mathrm{N}=311)\end{array}$ & $\begin{array}{l}\text { Normal discharge group } \\
\qquad(\mathrm{N}=193)\end{array}$ & p-value \\
\hline Drinking (\%) & & & & $<0.001$ \\
\hline Yes & $256(50.8)$ & $137(44.1)$ & $119(61.7)$ & \\
\hline No & $124(24.6)$ & $74(23.8)$ & $50(25.9)$ & \\
\hline Unmeasured (no response) & $124(24.6)$ & $100(32.2)$ & $24(12.4)$ & \\
\hline Psychiatric disease history (\%) & & & & $<0.001$ \\
\hline Yes & $194(38.5)$ & $120(38.6)$ & $74(38.3)$ & \\
\hline No & $150(29.8)$ & $70(22.5)$ & $80(41.5)$ & \\
\hline Unmeasured (no response) & $160(31.7)$ & $121(38.9)$ & $39(20.2)$ & \\
\hline Method of suicidal attempt (\%) & & & & 0.015 \\
\hline Ingested poisoning & $283(56.2)$ & $161(51.8)$ & $122(63.2)$ & \\
\hline Briquette gas poisoning & $36(7.1)$ & $25(8.0)$ & $11(5.7)$ & \\
\hline Hanging & $24(4.8)$ & $19(6.1)$ & $5(2.6)$ & \\
\hline Cutting wrist/pun* & $144(28.6)$ & $98(31.5)$ & $46(23.8)$ & \\
\hline Death-leaps & $12(2.4)$ & $4(1.3)$ & $8(4.1)$ & \\
\hline Others & $5(1.0)$ & $4(1.3)$ & $1(0.5)$ & \\
\hline Acknowledgment about suicidal attempt (\%) & & & & $<0.001$ \\
\hline Yes & $267(53.0)$ & $145(46.6)$ & $122(63.2)$ & \\
\hline No & $42(8.3)$ & $20(6.4)$ & $22(11.4)$ & \\
\hline Unmeasured (no response) & $195(38.7)$ & $146(46.9)$ & $49(25.4)$ & \\
\hline Plan about suicidal attempt (\%) & & & & $<0.001$ \\
\hline Yes & $46(9.1)$ & $21(6.8)$ & $25(13.0)$ & \\
\hline No (on impulse) & $271(53.8)$ & $145(46.6)$ & $126(65.3)$ & \\
\hline Unmeasured (no response) & $187(37.1)$ & $145(46.6)$ & $42(21.8)$ & \\
\hline
\end{tabular}

Data were reported as frequency (percentage). p-values were calculated by chi-square test or Fisher's exact test as appropriate. *punctured wound

Table 3. Variables of suicidal attempt related outcome per type of discharge

\begin{tabular}{lccc}
\hline \multicolumn{1}{c}{ Variable } & $\begin{array}{c}\text { Total } \\
(\mathrm{N}=504)\end{array}$ & $\begin{array}{c}\text { Voluntary discharge group } \\
(\mathrm{N}=311)\end{array}$ & $\begin{array}{c}\text { Normal discharge group } \\
(\mathrm{N}=193)\end{array}$ \\
\hline Awareness condition (\%) & & & $126(65.3)$ \\
Alert & $348(69.0)$ & $222(71.4)$ & $31(16.1)$ \\
Verbal & $85(16.9)$ & $54(17.4)$ & $32(16.6)$ \\
Pain & $62(12.3)$ & $30(9.6)$ & $4(2.1)$ \\
Unresponsive & $9(1.8)$ & $5(1.6)$ & $62(32.1)$ \\
Medical severity (\%) & & $182(58.5)$ & $72(37.3)$ \\
Low & $244(48.4)$ & $89(28.6)$ & $59(30.6)$ \\
Moderate & $161(31.9)$ & $40(12.9)$ & 40.001 \\
High & $99(19.6)$ & & $40(20.7)$ \\
Suicidal attempt plan in future $(\%)$ & & $40(12.9)$ & $94(48.7)$ \\
Yes & $80(15.9)$ & $66(21.2)$ & $59(30.6)$ \\
No & $160(31.7)$ & $205(65.9)$ & $<0.001$ \\
Unmeasured & $264(52.4)$ & & \\
\hline
\end{tabular}

Data were reported as frequency (percentage). p-values were calculated by chi-square test or Fisher's exact test as appropriate 
department or hospital (Table 4, Figure 1). The percentage of patients who agreed to psychiatric treatment was $64.0 \%$ $(199 / 311)$ in the VDG and 69.9\% (135/193) in the NDG. Both groups showed a higher percentage $>60 \%$, but there was no significant difference between the two groups (Table
4, Figure 1). However, the percentage of patients who refused psychiatric treatment was $29.6 \%(92 / 311)$ in the VDG, which was more than twice as high as $12.4 \%(24 / 193)$ in the NDG (Table 4, Figure 1). The percentage of patients who agreed to case management was $68.4 \%(132 / 193)$ in the NDG, which

Table 4. Treatment modality per type of discharge

\begin{tabular}{|c|c|c|c|c|}
\hline Variable & $\begin{array}{c}\text { Total } \\
(\mathrm{N}=504)\end{array}$ & $\begin{array}{l}\text { Voluntary discharge group } \\
\qquad(\mathrm{N}=311)\end{array}$ & $\begin{array}{l}\text { Normal discharge group } \\
\qquad(\mathrm{N}=193)\end{array}$ & p-value \\
\hline Medical request to NP (\%) & & & & $<0.001$ \\
\hline Request & $334(66.3)$ & $199(64.0)$ & $135(69.9)$ & \\
\hline Patient refusal & $116(23.0)$ & $92(29.6)$ & $24(12.4)$ & \\
\hline No request & $54(10.7)$ & $20(6.4)$ & $34(17.6)$ & \\
\hline Case management service (\%) & & & & $<0.001$ \\
\hline Agreement & $238(47.2)$ & $106(34.1)$ & $132(68.4)$ & \\
\hline Refusal & $70(13.9)$ & $42(13.5)$ & $28(14.5)$ & \\
\hline Unmeasured & $196(38.9)$ & $163(52.4)$ & $33(17.1)$ & \\
\hline Linkage of community service (\%) & & & & $<0.001$ \\
\hline Complete & $60(11.9)$ & $24(7.7)$ & $36(18.7)$ & \\
\hline Unmeasured & $444(88.1)$ & $287(92.3)$ & $157(81.3)$ & \\
\hline Psychiatric treatment after discharge (\%) & & & & $<0.001$ \\
\hline Outpatient visit & $155(30.8)$ & $45(14.5)$ & $110(57.0)$ & \\
\hline Protection ward & $1(0.2)$ & $0(0.0)$ & $1(0.5)$ & \\
\hline No therapy & $96(19.0)$ & $65(20.9)$ & $31(16.1)$ & \\
\hline Unmeasured & $252(50.0)$ & $201(64.6)$ & $51(26.4)$ & \\
\hline Place when discharging (\%) & & & & $<0.001$ \\
\hline Emergency room & $279(55.4)$ & $242(77.8)$ & $37(19.2)$ & \\
\hline General ward & $225(44.6)$ & $69(22.2)$ & $156(80.8)$ & \\
\hline
\end{tabular}

Data were reported as frequency (percentage). p-values were calculated by chi-square test or Fisher's exact test as appropriate. NP: neuropsychiatry

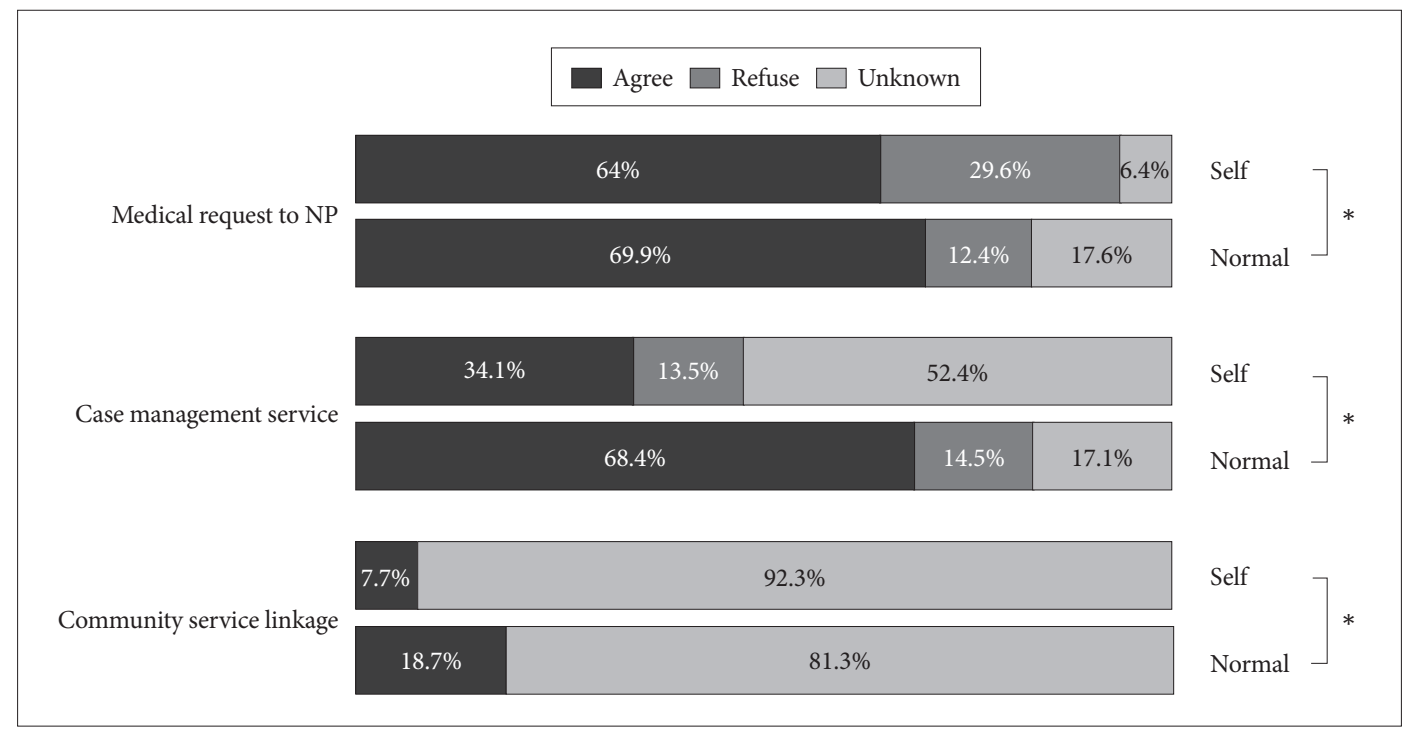

Figure 1. Treatment modality per type of discharge. ${ }^{*} p<0.05$. Self: voluntary discharge group, Normal: normal discharge group, NP: neuropsychiatry. 
was more than twice as high as $34.1 \%(106 / 311)$ in the VDG (Table 4, Figure 1). The percentage of patients who did not have information about their follow-up intervention in the VDG was $52.4 \%$ (163/311), which was more than three times as high as $17.1 \%$ (33/193) in the NDG (Table 4, Figure 1). The percentage of patients who visited the outpatient clinic (psychotherapy after discharge) was $57.0 \%(110 / 193)$ in the NDG, which was approximately four times as high as $14.5 \%(45 / 311)$ in the VDG (Table 4, Figure 2). The percentage of discharges from a hospital room was $80.8 \%(156 / 193)$ in the NDG, which was more than three times higher than the 22.2\% (69/311) in the VDG (Table 4). In contrast, the percentage of patients discharged from the emergency department was $77.8 \%(242 / 311)$ in the VDG, which was more than four times as high [19.2\% (37/193)] in the NDG (Table 4).

\section{The frequencies of emergency department voluntary discharge patients according to the visit and discharge time differences}

The total number of emergency department VDG patients was 242 (Table 4 and 5). Of these, 43 patients (17.8\%) visited

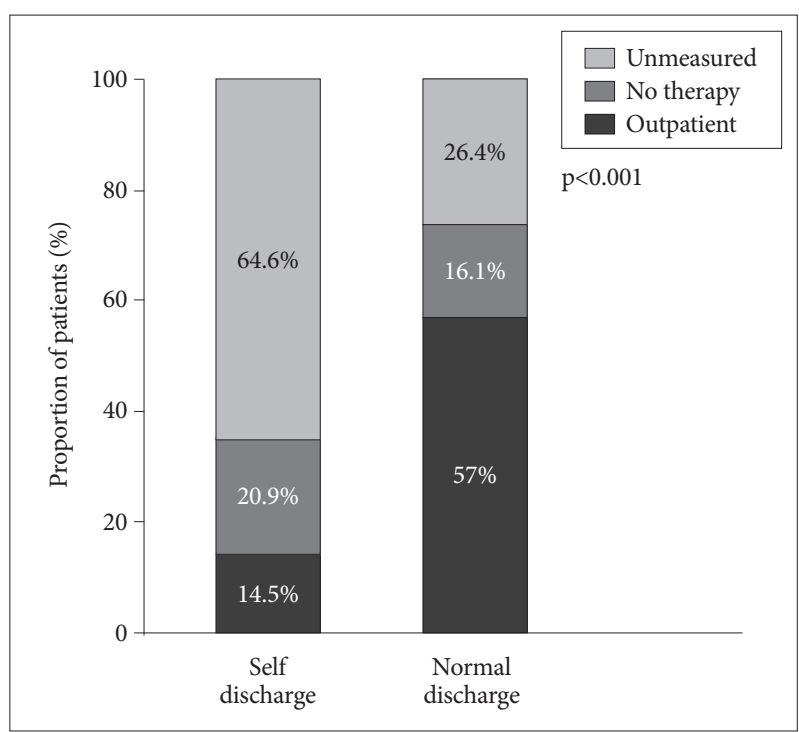

Figure 2. Psychiatric treatment after discharge. Self discharge: voluntary discharge group, Normal discharge: normal discharge group. the emergency department from 8:00 am to 17:00 pm when direct case management service in the emergency department was available (Table 5). In contrast, 185 patients $(82.2 \%)$ visited the emergency department from 17:00 pm to 8:00 am, when direct case management service in the emergency department was unavailable (Table 5). Eighty-five patients (35.1\%) were discharged from the emergency department from 8:00 am to 17:00 pm, when direct case management service in the emergency department was available (Table 5). In contrast, 157 patients (64.9\%) were discharged from the emergency department from 17:00 pm to 8:00 am, when direct case management service in the emergency department was unavailable (Table 5).

\section{Voluntary discharge outcomes according to univariate logistic regression analyses}

In addition to treatment-related variables, univariate logistic regression was performed on variables that were significant using the chi-square test for baseline characteristics, variables related to suicide attempts, and suicide attempt-related outcome variables (Table 6). There were significant differences in age, level of education, marital status, physical status, drinking status, psychiatric disease history, method of suicidal attempt, acknowledgement about the suicidal attempt, plans for future suicidal attempts, and the medical severity for voluntary discharges $(\mathrm{p}<0.05)$ (Table 6).

\section{Voluntary discharge outcomes according to multivariate logistic regression analyses}

The level of education, physical status, psychiatric disease history, and medical severity were significant $(\mathrm{p}<0.05)$ using multivariate logistic regression analyses of variables that were significant using univariate logistic tests for voluntary discharge (Table 7). The OR of the no response group regarding their level of education increased by 3.32 times compared with college-educated or higher patients (reference group) for voluntary discharge $(\mathrm{p}=0.004)$ (Table 7). Patients who did not respond to the physical status question had a 2.22 times higher increase in the OR compared with the healthy group for voluntary discharge $(\mathrm{p}=0.009)$ (Table 7$)$. Patients with a psychiatric disease history had an OR 1.62 times higher than pa-

Table 5. Frequencies of emergency department voluntary discharge patients according to visit and discharge time difference

\begin{tabular}{ccc}
\hline $\begin{array}{c}\text { Total emergency department voluntary discharge group } \\
(\mathrm{N}=242)\end{array}$ & $\begin{array}{c}\text { Time } \mathrm{A}^{*} \\
(8: 00 \text { am to 17:00 } \mathrm{pm})\end{array}$ & $\begin{array}{c}\text { Time } \mathrm{B}^{\dagger} \\
(17: 00 \mathrm{pm} \text { to 8:00) }\end{array}$ \\
\hline Emergency department (\%) & & $185 / 242(82.2)$ \\
Visit group & $43 / 242(17.8)$ & $157 / 242(64.9)$ \\
Discharge group & $85 / 242(35.1)$ & \\
\hline
\end{tabular}

${ }^{*}$ time from 8:00 am to 17:00 pm when direct case management service in emergency department was available, ${ }^{\dagger}$ time from 17:00 pm to 8:00 am when direct case management service in emergency department was unavailable 
Table 6. Univariate logistic regression analysis for voluntary discharge in subjects attempting suicide

\begin{tabular}{|c|c|c|}
\hline \multirow{2}{*}{ Variable } & \multicolumn{2}{|l|}{ Univariate } \\
\hline & OR (95\% CI) & $\mathrm{p}$-value \\
\hline \multicolumn{3}{|l|}{ Sex } \\
\hline Male & 1 & \\
\hline Female & $1.34(0.92-1.95)$ & 0.125 \\
\hline \multicolumn{3}{|l|}{ Age (year) } \\
\hline$<60$ & 1 & \\
\hline$\geq 60$ & $0.45(0.27-0.75)$ & 0.002 \\
\hline \multicolumn{3}{|l|}{ Educational level } \\
\hline$\geq$ College & 1 & \\
\hline High school or less & $1.22(0.56-2.8)$ & 0.619 \\
\hline No response & $5.86(2.73-13.21)$ & $<0.001$ \\
\hline \multicolumn{3}{|l|}{ Marital status } \\
\hline Married & 1 & \\
\hline Unmarried & $1.72(1.12-2.65)$ & 0.013 \\
\hline Married but without spouse & $1.95(1.24-3.09)$ & 0.004 \\
\hline \multicolumn{3}{|l|}{ Physical status } \\
\hline Healthiness & 1 & \\
\hline Disease/disability & $0.7(0.45-1.07)$ & 0.097 \\
\hline Unmeasured & $4.55(2.75-7.73)$ & $<0.001$ \\
\hline \multicolumn{3}{|l|}{ Drinking } \\
\hline No & 1 & \\
\hline Yes & $0.78(0.5-1.2)$ & 0.258 \\
\hline Unmeasured & $2.82(1.6-5.05)$ & $<0.001$ \\
\hline \multicolumn{3}{|l|}{ Psychiatric disease history } \\
\hline No & 1 & \\
\hline Yes & $1.85(1.2-2.86)$ & 0.005 \\
\hline Unmeasured & $3.55(2.2-5.79)$ & $<0.001$ \\
\hline \multicolumn{3}{|l|}{ Method of suicidal attempt } \\
\hline (Gas) Poisoning & 1 & \\
\hline Hanging/stang & $1.64(1.11-2.45)$ & 0.015 \\
\hline Others & $0.64(0.23-1.7)$ & 0.364 \\
\hline \multicolumn{3}{|c|}{ Acknowledgement about suicidal attempt } \\
\hline Yes & 1 & \\
\hline No & $0.76(0.4-1.47)$ & 0.42 \\
\hline Unmeasured & $2.51(1.68-3.77)$ & $<0.001$ \\
\hline \multicolumn{3}{|l|}{ Plan about suicidal attempt } \\
\hline Yes & 1 & \\
\hline No (on impulse) & $1.37(0.73-2.59)$ & 0.325 \\
\hline Unmeasured & $4.11(2.1-8.14)$ & $<0.001$ \\
\hline \multicolumn{3}{|l|}{ Medical lethality } \\
\hline High & 1 & \\
\hline Moderate & $1.82(1.1-3.04)$ & 0.02 \\
\hline Low & $4.33(2.65-7.14)$ & $<0.001$ \\
\hline
\end{tabular}

OR with $95 \% \mathrm{CI}$, p-values were calculated by univariate logistic regression analysis. OR: odds ratio, CI: confidence interval
Table 7. Multivariate logistic regression analysis for voluntary discharge in patients attempting suicide

\begin{tabular}{lcc}
\hline \multirow{2}{*}{ Variable } & \multicolumn{2}{c}{ Multivariate } \\
\cline { 2 - 3 } Educational level & OR $(95 \% \mathrm{CI})$ & $\mathrm{p}$-value \\
$\quad$ College & 1 & \\
High school or less & $1.13(0.5-2.62)$ & 0.776 \\
No response & $3.32(1.47-7.82)$ & 0.004 \\
Physical status & 1 & \\
Healthiness & $0.67(0.42-1.06)$ & 0.086 \\
Disease/disability & $2.22(1.23-4.07)$ & 0.009 \\
Unmeasured & & \\
Psychiatric disease history & 1 & 0.046 \\
No & $1.62(1.01-2.61)$ & 0.54 \\
Yes & $1.2(0.67-2.15)$ & \\
Unmeasured & & 0.412 \\
Medical lethality & 1 & 0.008 \\
High & $1.26(0.73-2.19)$ \\
Moderate & $2.1(1.21-3.65)$ & \\
Low & & \\
\hline OR & & \\
\hline
\end{tabular}

OR with 95\% CI, p-values were calculated by multivariate logistic regression analysis. OR: odds ratio, CI: confidence interval

tients without a psychiatric disease history $(\mathrm{p}=0.046)$ (Table 7). The OR of the low medical severities group was 2.1 times higher than that of patients with high medical severities ( $\mathrm{p}=$ 0.008) (Table 7).

\section{DISCUSSION}

The voluntary discharge of patients accounts for approximately $2 \%$ of all hospital discharges, which results in a quality problem for health care. ${ }^{4}$ In a general medical service study, the percentage of rehospitalizations in the voluntary discharge patient group within 15 days of the same diagnosis was always seven times higher than that of the normally treated group. ${ }^{4,5}$ In the present study, we classified factors affecting voluntary and normal discharge of suicide attempt patients, including the emergency department and hospital admission [ward or intensive care unit (ICU)] as basic characteristics (demographic variables), suicide attempt-related variables, suicide attemptrelated outcome variables per type of discharge, and treatment modality variables per type of discharge. Of these, demographic variables, suicide attempt-related variables, and suicidal attempt-related outcome variables per type of discharge were variables for predicting the voluntary discharge of patients. However, the category of treatment modality variables per type of discharge was variable due to the voluntary discharge of the patient. 
Regarding demographic variables, the percentage of VDG patients was significant depending on the age, level of education, marital status, and physical health. The percentage of voluntary discharges of patients $\leq 20$ years of age was high, and the percentage of normal discharges was high in patients $\geq 50$ years of age. It was found that the younger the age, the higher the probability of voluntary discharge. In the case of elderly patients $\geq 50$ years of age, the patient's or caregiver's concerns about the patient's physical health may have caused an increase in the percentages of admissions and normal discharges. The percentage of voluntary discharge from the married group was the lowest in the VDG, which was presumed to be influenced by the emotional intervention of the spouse.

In the case of alcoholic patients related to suicide attempts, the percentage of drinkers was twice as high as that of nondrinkers in the VDG when the unmeasured patients were excluded. This was consistent with the finding that most past retrospective studies have consistently involved decisions to use a person's discharge note if there was a drug or alcohol problem. ${ }^{4}$ The most frequent method of suicidal attempt was ingested poisoning, including briquette gas poisoning, and wrist cutting/puncture wound was the next most frequent. The percentage of ingested poisoning, including briquette gas poisoning, was also the highest, and wrist cutting/puncture wound was the next most frequent in the VDG. In a Korean study of adolescents aged 10-19 years, ingested poisoning was the most common method of attempting suicide, while hanging and falling were the most lethal suicide attempts, and the percentage of voluntary discharge from the emergency department was $22.8 \% .{ }^{6}$ Patients with a psychiatric history were more likely to be discharged against medical advice. This result is partially consistent with a study reporting that the discharge of a patient was most commonly predicted from patient factors, such as pessimistic attitudes toward treatment; aggressive, destructive, and anti-social behavior; multiple voluntary discharges from previous hospitalizations; a young age; male; unmarried; and an accompanying personality disorder or substance abuse diagnosis. ${ }^{7}$ Other studies have also reported that the percentage of patients discharged voluntarily from the psychiatric group ranged from 3-51\% (average, 17\%), which was much higher than that of the medical group. ${ }^{7}$ In the NDG involving ingested poisoning, medical treatment was often necessary, mainly because the ingested poison involved sleeping pills or psychiatric drugs, leading to a decreased consciousness that made the patient unable to be discharged early. Awareness condition was not significantly associated with the voluntary discharge of the patient, in contrast to lower medical severity, which resulted in a significantly higher percentage of voluntary discharges. The unmeasured group with future plans for a suicide attempt had the highest percentage of patients in the VDG. In these patients, the non-responders were judged to be untreated patients, and because of the highest percentage of voluntary discharge of untreated patients, it was thought that a 24 -h care system was needed to separately manage suicide attempters in the hospital. Suicide attempters were mostly impulsive. Excluding non-responders, impulsive patients were more likely to be voluntarily discharged.

The variables related to treatment, including psychiatric referral, consent, and intervention of a case management service; link with community service; post-discharge psychiatric treatment; and the location of the discharge (emergency department, general ward, or the ICU) were significantly associated with the type of discharge of the suicide attempter. Of the total of 311 voluntary discharge patients, 242 (77.8\%) were discharged from the emergency department. The percentage of patients who refused psychiatric consultation in the VDG was more than twice that in the group who refused psychiatric consultation in the NDG. This was consistent with previous results from Holden et al. ${ }^{8}$ that indicated the need for early psychiatric intervention as there was a tendency for medical patients to be less voluntarily discharged. The percentage of patients who agreed to psychiatric consultation in the VDG was similar to that of the NDG. In contrast, the percentage of the group who agreed to case management services in the NDG was more than twice as high as that in the VDG. The percentage of patients who agreed to psychiatric outpatient visits in the NDG was four times higher than that of patients in the VDG. These results indicated that the consent of psychiatric consultation alone did not have a significant effect on the patient's discharge pattern, but it was found that when the case management service was linked, it increased the percentage of connections among normal discharge, community links, and psychiatric outpatient visits. These results indicated that most of the normally discharged patients were discharged from entering the hospital ward, and that the case manager could easily intervene in a stable state, so that the percentage of agreement was high. However, in cases of voluntary discharge, the agreement percentage of the post-management service was lower than that of the NDG. In particular, when a patient was discharged voluntarily from the emergency department, there were many cases in which the case management team member was not able to intervene during the off-duty hours, ranging from 5:00 pm to 8:00 am. Based on these findings, it is important for the national government to establish and fund a system to manage suicide attempters for $24 \mathrm{~h}$.

The percentages of the unmeasured or non-responder group involving variables such as the level of education, physical status, drinking status, psychiatric disease history, hospitalization history due to suicide attempts, plans for future suicide attempts, case management services, link with community 
services, and psychotherapy after discharge in the VDG were higher than those in the NDG. In VDG patients, there was a tendency not to accurately convey the condition of the patient or the coping process of the suicide attempter in the hospital care system could not be properly determined.

Whether the hospital had a psychiatric closed ward was also considered as a contributing factor for voluntary discharge of suicide attempters. In most of the admitted suicide attempters, in the course of the retrospective chart review of this study, after medical or surgical treatment was terminated, the patient was referred to a psychiatric department for treatment. In the course of this process, it was frequently found that patients and their caregivers were not compliant with the procedures and were discharged voluntarily from the hospital when they were told that they needed to be transferred to other hospitals with closed wards.

Interviews with the case management team staff at this research institute indicated that this team needed active participation by the medical staff. This meant that when the case management team was later contacted, the patient and caregiver showed a favorable attitude that led to post-management and community links with positive results.

Based on the results of the multivariate logistic regression analyses, the OR of the no response group relative to the college graduation or higher group, the physical status of the no response group relative to the healthy group, the psychiatric disease history group relative to the no psychiatric disease history group, and the low medical severity group relative to the high medical group were proportional to the percentages of voluntary discharges. It is anticipated that knowing the characteristics of the variables mentioned above and studying past investigations will help to reduce the voluntary discharge of patients from the hospital. Some studies have suggested that early detection, discussions, and counseling for communication are the preferred methods for reducing the percentage of discharges among patients with a history of drug or alcohol abuse. ${ }^{4}$ Targum et al. ${ }^{9}$ reported that there was an approximate 30\% decrease in all voluntary discharges of psychiatric inpatients from private hospitals after appointing a nurse as a patient spokesperson, whose responsibility was to resolve the patient's concerns about hospitalization, point out the fears, and alert the hospital staff about these concerns and fears. Alfandre suggested that to prevent voluntary discharges, the patient should be interviewed to recognize mental factors including substance addiction. ${ }^{4}$ Another study reported that physicians who started their care with a suspicious attitude tended to meet with patients in uncomfortable conditions and were likely to terminate treatment under poor conditions. All patients should therefore be granted empathy regardless of the diagnosis, but should also be entitled to a more accurate and effective patient assessment. ${ }^{10,11}$ Studies have shown that when a medical officer is angered when dealing with a difficult type of patient with persistent physical complaints, increased anger and insomnia may be associated with dependence or loss of disinhibition that prevents communication, which in turn distracts the patient and, in the worst cases, dismisses the caregiver, leading to voluntary discharge without adequate treatment of the patient. ${ }^{4}$ However, some studies have shown that if the medical staff has a good understanding of the anger-like feelings that naturally occur while they are in contact with these demanding patients, they can better refocus their discussions with the patient, and a psychiatric interview can be helpful. ${ }^{4}$ Another study reported that patients who decide to voluntarily discharge for personal reasons may prioritize financial interests rather than concern for their health and should be respected if it is an informed decision. ${ }^{4}$ Levy et al. ${ }^{12}$ suggested that if the medical staff was unable to prevent voluntary discharge of their patients, they should perform three necessary steps that will absolve the medical staff from legal responsibilities. First, the medical staff should confirm that the patient is in a condition, with a normal mental status, to refuse treatment. Second, the medical staff should explain all potential risks to the patient and patient guardian or caregiver. Finally, the medical staff should take steps to retain the patient's discharge with the medical advice consent in a suitable chart.

This study was conducted based on a retrospective medical record survey at a single hospital, and it occurred during a specific period of time, so there was a possibility that there was selection bias and incomplete data discrimination. For example, patients recorded as "ordinary type" may have involved local enrollment or company enrollment, but it was impossible to distinguish between them. To overcome these limitations, future prospective multidisciplinary studies will be necessary.

Because suicide is caused by multiple causes, various forms of follow-up care are needed after discharge, and resources such as case management and community centers should be available.

In conclusions, three hundred eleven patients (61.7\%) of the total 504 suicide attempters were discharged from the hospital. The non-response group relative to the college graduation or higher group, the physical status of the non-response group relative to the healthy group, the psychiatric disease history group relative to the no psychiatric disease history group, and the low medical severity group relative to the high medical severity group were all proportional. The VDG had a very low percentage of post-discharge intervention in the ongoing treatment methods of psychiatric outpatients, case management, and community links. Although 
case management services prevented and reduced the discharge of suicide attempters and increased the link between community and outpatient mental health services after discharge, it was not known whether at least $80 \%$ of the total suicide attempters were still linked to community services. Based on these results, further aspects of national supportive measures, including strengthening case management services, should be considered to reduce voluntary discharge of suicide attempters.

\section{REFERENCES}

1. Ministry of Health \& Welfare, Korea Suicide Prevention Center. 2016 White Book. Seoul: Korea Suidice Prevention Center; 2016.

2. Silverman MM, Berman AL, Sanddal ND, O'carroll PW, Joiner TE. Rebuilding the tower of babel: a revised nomenclature for thestudy of suicide and suicidal behaviors Part 1: Background, rationale, and methodoloy. Suicide Life Threat Behav 2007;37:248-263.

3. Durkheim E. Suicide: A Study in Sociology. London and New York: Routledge; 2002.

4. Alfandre DJ. "I'm going home": discharges against medical advice. Mayo Clin Proc 2009;84:255-260.
5. Hwang SW, Li J, Gupta R, Chien V, Martin RE. What happens to patients who leave hospital against medical advice? CMAJ 2003;168:417420.

6. Jung JH, Kim DK, Jung JY, Lee JH, Kwak YH. Risk factors of discharged against medical advice among adolescents self-inflicted injury and attempted suicide in the Korean emergency department. J Korean Med Sci 2015;30:1466-1470.

7. Brook M, Hilty DM, Liu W, Hu R, Frye MA. Discharge against medical advice from inpatient psychiatric treatment: a literature review. Psychiatr Serv 2006;57:1192-1198.

8. Holden P, Vogtsberger KN, Mohl PC, Fuller DS. Patients who leave the hospital against medical advice: the role of the psychiatric consultant. Psychosomatics 1989;30:396-404.

9. Targum SD, Capodanno AE, Hoffman HA, Foudraine C. An intervention to reduce the rate of hospital discharges against medical advice. Am J Psychiatry 1982;139:657-659.

10. Passik SD, Byers K, Kirsh KL. Empathy and the failure to treat pain. Palliat Support Care 2007;5:167-172.

11. Gallagher RM. Empathy: a timeless skill for the pain medicine toolbox. Pain Med 2006;7:213-214.

12. Levy F, Mareiniss DP, Iacovelli C. The importance of a proper againstmedical-advice(AMA) discharge: how signing out AMA may create significant liability protection for providers. J Emerg Med 2012;43:516520. 